2013-10-12

\title{
Sensitivity Enhancement for a Multimode Fiber Sensor with an Axisymmetric Metal Grating Layer
}

\author{
Youqiao Ma \\ Technological University Dublin \\ Gerald Farrell \\ Technological University Dublin, gerald.farrell@tudublin.ie \\ Yuliya Semenova \\ Technological University Dublin, yuliya.semenova@tudublin.ie
}

See next page for additional authors

Follow this and additional works at: https://arrow.tudublin.ie/engscheceart

\section{Recommended Citation}

Ma, Y., Farrell, G., Semenova, Y., Chan, H., Zhang, H. and Wu, Q. Sensitivity Enhancement for a Multimode Fiber Sensor with an Axisymmetric Metal Grating Layer. Photonics and Nanostructures - Fundamentals and Applications. In Press, Accepted Manuscript, Available online 22 August 2013. doi:10.1016/ j.photonics.2013.08.001

This Article is brought to you for free and open access by the School of Electrical and Electronic Engineering at ARROW@TU Dublin. It has been accepted for inclusion in Articles by an authorized administrator of ARROW@TU Dublin. For more information, please contact arrow.admin@tudublin.ie, aisling.coyne@tudublin.ie, gerard.connolly@tudublin.ie.

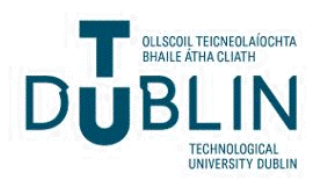




\section{Authors}

Youqiao Ma, Gerald Farrell, Yuliya Semenova, Hauping Chan, Hongzhou Zhang, and Qiang wu 


\title{
Sensitivity Enhancement for a Multimode Fiber Sensor with an Axisymmetric Metal Grating Layer
}

\author{
Youqiao Ma ${ }^{1}$, Gerald Farrell ${ }^{1}$, Yuliya Semenova ${ }^{1}$, Hau Ping Chan ${ }^{2}$, Hongzhou Zhang, ${ }^{3,4}$ and Qiang Wu ${ }^{1}$ \\ ${ }^{1}$ Photonics Research Center, School of Electronic and Communications Engineering, Dublin Institute of Technology, Kevin Street, \\ Dublin 8, Ireland \\ ${ }^{2}$ Department of Electronic Engineering, City University of Hong Kong, Hong Kong, China \\ ${ }^{3}$ School of Physics, Trinity College Dublin, Dublin 2, Ireland \\ ${ }^{4}$ CRANN, Trinity College, Dublin 2, Ireland
}

\begin{abstract}
This paper proposes a novel design for a surface plasmon resonance (SPR) fiber sensor with an axisymmetric sub-wavelength metal grating layer. The relationship between the sensor performance (the sensitivity $S$ and the quality factor $Q$ of the SPR dip) and the characteristic parameters are investigated. Numerical simulation results show that the proposed sensor can achieve a maximum sensitivity of $13000 \mathrm{~nm} / \mathrm{RIU}$ (refractive index unit) for a refractive index range from 1.3 to 1.4.
\end{abstract}

\section{Introduction}

Over the last two decades, surface plasmon resonance (SPR), which refers to the excitation of surface plasmon polaritons (SPPs) at the interface between a dielectric (i.e. gas, solid or liquid) and a metal (e.g. gold or silver), has become a useful and reliable technique for chemical and biochemical sensing applications [1-3]. Traditional SPR sensors are based on the so-called Kretschmann configuration, where a thin metal film is deposited on the base of a coupling prism [4]. For such prism-based SPR sensors, Homola provides a comprehensive overview of developments in the past 20 years and numerous designs for novel SPR biosensors [5]. However, a prism-based SPR configuration has a number of disadvantages, such as bulky size and complex operation and in addition real time/remote sensing is difficult to implement. In order to overcome these disadvantages, SPR-based optical fiber sensors have attracted significant interest [6-9]. A fiber based sensing configuration allows for a compact sensing element and sample volume, the capacity for remote sensing and the potential for disposable miniaturized sensing devices. In the past decade, various fiber based SPR sensors have been proposed and demonstrated, including the use of either single mode fibers [10] or multimode fibers [11].

Recently, several fiber based SPR sensors have been presented such as a multimode fiber coated with bimetallic layers (silver and gold, with gold as the outer layer), a hetero-core fiber with $\mathrm{Au} / \mathrm{Ta}_{2} \mathrm{O}_{5} / \mathrm{Pd}$ multi-layers, and a multimode fiber covered with a nano-particle metal film, which were both studied theoretically based on the attenuated total reflection (ATR) method and multi-layer model [12-15]. Among these studies, the effects of the parameters and characteristics of the optical fiber and metal layer on the sensor sensitivity have been investigated in detail, for example recently the relationships between the resonance wavelength and the thickness of the metal layer and sensing layer were investigated [16].

In this paper, a novel fiber SPR-based biosensor coated with an axisymmetric sub-wavelength metal grating layer is proposed and investigated theoretically. The paper is organized as follows: In section 2, the theoretical background and detailed relative numerical model in the form of multilayer model are provided. In section 3, the dependence of the device parameters (i.e. the metal grating layer thickness, the metal grating period, the grating length and the diameter of the fiber core) on the refractive index (RI) sensitivity $S$ and the quality factor $Q$ of the SPR dip are evaluated numerically. The conclusions are provided in section 4 .

\section{Theoretical formalism}

We initially consider the attenuated total reflection (ATR) method with a Kretschmann configuration (Fig.1) [5] as this forms the basis of the theoretical model for the fiber SPR-based sensor proposed in this 
paper. As shown in Fig.1, the structure comprises a coupling prism with a high refractive index (RI), a metal layer and a sensing sample. If the resonance condition is satisfied, that is the wave-vector of the incident light matches that of the SPPs, the output spectrum demonstrates a dip located at the resonance wavelength. The resonance wavelength is highly sensitive to the RI variations of the sensing sample, i.e. the resonance wavelength $\left(\lambda_{\mathrm{R}}\right)$ shifts by $\delta \lambda_{\mathrm{R}}$ when the RI of the sensing sample alters by $\delta n$. In this respect two parameters can be introduced to describe the sensor's performance: the sensitivity $(S)$ and the quality factor $(Q)$ of the resonance dip which can be written as:

where $\Delta \lambda$ is $3 \mathrm{~dB}$ bandwidth.

$$
S=\frac{\delta \lambda_{R}}{\delta n}, Q=\frac{\lambda_{R}}{\Delta \lambda}
$$

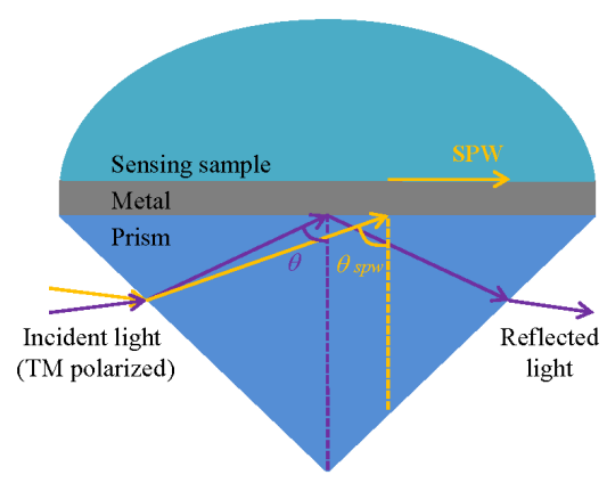

Fig. 1 Kretschmann configuration of a prism-based SPR sensing system.

To calculate the transmission of TM-polarized incident light through the structure, a multilayer model (Fig. 2) is used. The layers are assumed to be stacked along the z-axis. Each layer is defined by a thickness of $h_{\mathrm{m}}$, permeability $\mu_{\mathrm{m}}$, dielectric constant $\varepsilon_{\mathrm{m}}$ and RI $n_{\mathrm{m}}$, where $m=2,3,--, N-1$.

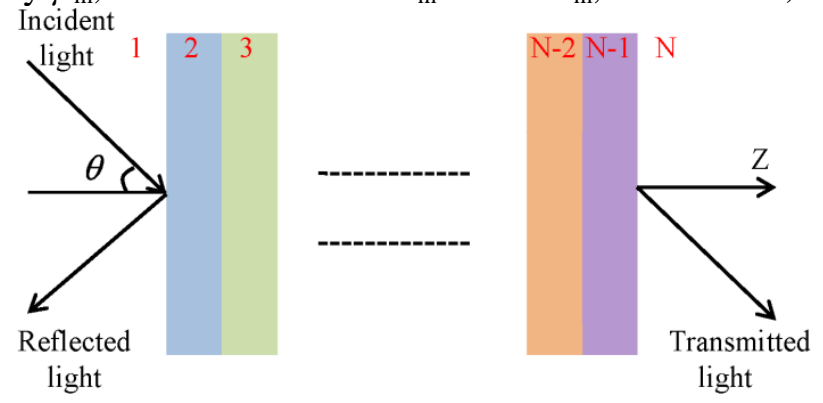

Fig. 2 Representation of the multilayer model. All the structural parameters and coordinate are shown in the figure.

The relationship of the tangential fields between the first boundary $Z_{1}$ and the final boundary $Z_{\mathrm{N}-1}$ is expressed as:

$$
\left[\begin{array}{l}
E_{1}^{/ /} \\
H_{1}^{/ /}
\end{array}\right]=M\left[\begin{array}{l}
E_{\mathrm{N}-1}^{/ /} \\
H_{\mathrm{N}-1}^{/ /}
\end{array}\right]
$$

where $E / /$ and $H^{/ /}$represent the tangential component of the electric and magnetic field at the boundary, respectively. Here $M$ is the characteristic matrix and is given by:

with

$$
M=\prod_{\mathrm{m}=2}^{\mathrm{N}-1} M_{m}=\left[\begin{array}{ll}
M_{11} & M_{12} \\
M_{21} & M_{22}
\end{array}\right]
$$

where

$$
M_{\mathrm{m}}=\left[\begin{array}{cc}
\cos \beta_{\mathrm{m}} & \frac{-i \sin \beta_{\mathrm{m}}}{q_{\mathrm{m}}} \\
-i q_{\mathrm{m}} \sin \beta_{\mathrm{m}} & \cos \beta_{\mathrm{m}}
\end{array}\right]
$$




$$
\beta_{\mathrm{m}}=\frac{2 \pi h_{\mathrm{m}}}{\lambda} \sqrt{\varepsilon_{\mathrm{m}}-\left(n_{1} \sin \theta\right)^{2}}, q_{\mathrm{m}}=\frac{\sqrt{\varepsilon_{\mathrm{m}}-\left(n_{1} \sin \theta\right)^{2}}}{\varepsilon_{\mathrm{m}}}
$$

Finally the reflectivity for the $\mathrm{N}$ layer stacked structure illuminated by a TM-polarized light is given as:

$$
R_{\mathrm{TM}}=\left|\frac{\left(M_{11}+M_{12} q_{\mathrm{N}}\right) q_{1}-\left(M_{21}+M_{22} q_{\mathrm{N}}\right)}{\left(M_{11}+M_{12} q_{\mathrm{N}}\right) q_{1}+\left(M_{21}+M_{22} q_{\mathrm{N}}\right)}\right|^{2}
$$

For the fiber-based SPR sensor, the prism is replaced by the silica core of a multimode fiber (Fig. 3). The cladding around the central region of the fiber is removed and coated with a sub-wavelength metal grating layer, which is assumed to be surrounded by the sensing sample with a RI of $n_{\mathrm{s}}$.

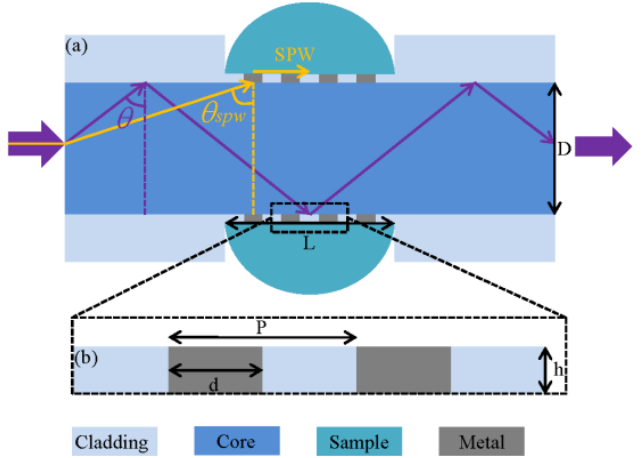

Fig. 3 (a) Schematic diagram for a Fiber-based SPR sensor with a sub-wavelength metal grating layer. (b) Illustration of the sub-wavelength metal grating layer. All the structural parameters are shown in the figure.

Based on the quasi-static limits (i.e. the grating-period-to-wavelength ratio $\mathrm{P} / \lambda \rightarrow 0$ ), the optical properties of a sub-wavelength grating are equivalent to a uniaxial homogeneous layer whose effective permittivity can be calculated by the effective medium theory (EMT) [17]. The effective dielectric function $\varepsilon_{\text {eff }}$ can be written as $\varepsilon_{\text {eff }}=\varepsilon_{\mathrm{D}}+\left(\varepsilon_{\mathrm{M}}-\varepsilon_{\mathrm{D}}\right) d / P$, where $\varepsilon_{\mathrm{D}}$ and $\varepsilon_{\mathrm{M}}$ are the permittivities of the dielectric and metal, respectively. The frequency-dependent complex relative permittivity of the metal (gold) is characterized by the Drude model [18] i.e. $\varepsilon_{\mathrm{M}}(\omega)=9.1-\omega_{\mathrm{p}} / \omega(\omega+i \gamma)$, where $\omega_{\mathrm{p}}\left(=1.2 \times 10^{16} \mathrm{rad} / \mathrm{s}\right)$, $\gamma\left(=1.2 \times 10^{14} \mathrm{rad} / \mathrm{s}\right)$ and $\omega$ represent the bulk plasma frequency, the damping frequency of the free electron oscillations and the angular frequency of the incident light, respectively.

Assuming the light is launched into one end of the fiber, the normalized transmitted power detected at another end of the fiber can be written as [19]:

where

$$
P_{T}=\frac{\int_{\theta_{\mathrm{cr}}}^{\pi / 2} R_{\mathrm{TM}}^{\mathrm{K}}\left(n_{\mathrm{co}}^{2} \sin \theta \cos \theta\right) /\left(1-n_{\mathrm{co}}^{2} \cos ^{2} \theta\right)^{2} d \theta}{\int_{\theta_{\mathrm{cr}}}^{\pi / 2}\left(n_{\mathrm{co}}^{2} \sin \theta \cos \theta\right) /\left(1-n_{\mathrm{co}}^{2} \cos ^{2} \theta\right)^{2} d \theta}
$$

$$
K=\frac{L}{D \tan \theta}, \theta_{\mathrm{cr}}=\sin ^{-1}\left(n_{\mathrm{cl}} / n_{\mathrm{co}}\right)
$$

In Eq. (8), $K$ is the number of ray reflections; $L$ and $D$ are the length of the sensing region and the fiber core diameter, respectively; $\theta$ is the angle of the ray normal to the core-metal interface; $n_{\mathrm{co}}\left(n_{\mathrm{co}}=1.457\right)$ and $n_{\mathrm{cl}}\left(n_{\mathrm{cl}}=1.4395\right)$ are the RI of the fiber core and cladding, respectively.

\section{Simulation results and discussions}

As shown in Fig. 3, a three-layer configuration (fiber core/sub-wavelength metal grating layer/sensing sample layer) are considered in our analysis. When the wave-vector of the incident light $\left(k_{\text {in }}\right)$ parallel to the core-metal grating interface matches that of SPPs $\left(k_{\mathrm{SP}}\right)$, a strong surface plasmon wave (SPW) is generated which results in a clear SPR dip in the corresponding transmission spectrum. The resonance condition can be expressed by the following equation [17]: 


$$
k_{\mathrm{SP}}=k_{\mathrm{in}} \sin \theta \pm k_{\mathrm{G}}=k_{\mathrm{in}} \sqrt{\frac{\varepsilon_{\mathrm{co}} \varepsilon_{\mathrm{eff}}}{\varepsilon_{\mathrm{co}}+\varepsilon_{\mathrm{eff}}}}+2 k_{\mathrm{in}} \frac{\varepsilon_{\mathrm{eff}} \varepsilon_{\mathrm{S}}}{\varepsilon_{\mathrm{eff}}^{2}-\varepsilon_{\mathrm{S}}^{2}} \frac{\varepsilon_{\mathrm{co}}-\sqrt{\varepsilon_{\mathrm{co}} \varepsilon_{\mathrm{eff}}+\varepsilon_{\mathrm{S}}\left(\varepsilon_{\mathrm{co}}-\varepsilon_{\mathrm{eff}}\right)}}{\varepsilon_{\mathrm{co}}+\sqrt{\varepsilon_{\mathrm{co}} \varepsilon_{\mathrm{eff}}+\varepsilon_{\mathrm{S}}\left(\varepsilon_{\mathrm{co}}-\varepsilon_{\mathrm{eff}}\right)}} \exp \left(2 i k_{\mathrm{in}} h \frac{\varepsilon_{\mathrm{eff}}}{\sqrt{\varepsilon_{\mathrm{eff}}+\varepsilon_{\mathrm{S}}}}\right)
$$

where $k_{\mathrm{G}}$ is the grating vector (i.e. $k_{\mathrm{G}}=2 \mathrm{~m} \pi / \mathrm{P}, m$ is an integer no less than zero), $\varepsilon_{\mathrm{co}}$ is the permittivity of the fiber core, and $\varepsilon_{\mathrm{s}}$ is the permittivity of the sensing sample.

The sensor's performance is evaluated based on the calculation model shown above. Fig. 4 (a) firstly depicts the normalized transmission spectra for different refractive indices of $n_{s}$. In the simulation, the structural parameters are set to be $L=20 \mathrm{~mm}, D=400 \mathrm{um}, d=10 \mathrm{~nm}, P=20 \mathrm{~nm}, \mathrm{~h}=30 \mathrm{~nm}$ and $\varepsilon_{\mathrm{D}}=$ $n_{\mathrm{cl}}^{2}$. From Fig.4 (a) we can see that as $n_{\mathrm{s}}$ increases, the SPR dip shifts toward a longer wavelength, which shows that RI changes for the sample surrounding the metal grating film can be monitored by measuring the shift of the resonance wavelength. To discuss the choice of a metal grating layer (i.e. $d=10 \mathrm{~nm}$ ) respect to a uniform metal layer (i.e. $d=P=20 \mathrm{~nm}$ ), Fig. 4 (b) shows the calculated sensitivities for both two cases. It confirms that as the RI of the sample increases the central resonance wavelength of two cases increase monotonically. Comparing these two cases, it is easy to see that the fiber-based SPR sensor with the metal grating layer shows a higher sensitivity than that of sensor with the uniform metal layer, which are demonstrated in the inset of Fig. 4(b). It shows that the proposed Fiber-based SPR sensor has an estimated sensitivity of $13000 \mathrm{~nm} / \mathrm{RIU}$ in the RI range from 1.395 to 1.40 which is much higher than that of $7500 \mathrm{~nm} / \mathrm{RIU}$ reported in [15] and $3892 \mathrm{~nm} / \mathrm{RIU}$ in the RI range from 1.333 to 1.353 which is comparable to that of $4262 \mathrm{~nm} / \mathrm{RIU}$ achieved in [2].
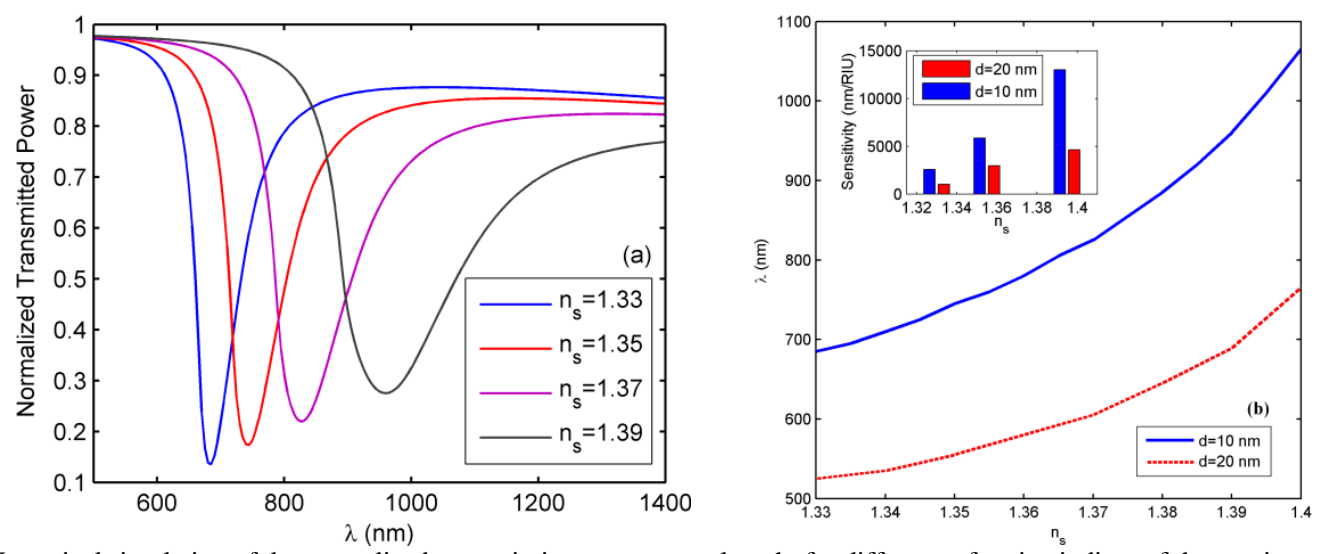

Fig. 4 (a) Numerical simulation of the normalized transmission versus wavelength for different refractive indices of the sensing sample. (b) Calculated central wavelength vs. the sensing sample refractive index and the sensitivity (inset).

In order to better understand the transmission properties of the Fiber-based SPR sensor, the spectral response of the structure with various characteristic parameters is studied. According to the resonance condition of Eq. (9), it can be shown that the resonance wavelength of sensor is related to the grating period of $P$ and the grating thickness of $h$. Fig. 5 (a) firstly depicts the normalized transmission spectra at different values of $P$. In this simulation, the structural parameters are $L=20 \mathrm{~mm}, D=400 \mathrm{um}, \mathrm{h}=30 \mathrm{~nm}$, $n_{\mathrm{s}}=1.36, d=10 \mathrm{~nm}$ and $\varepsilon_{\mathrm{D}}=n_{\mathrm{cl}}^{2}$. From Fig. 5 (a) one can see that the spectral response is dependent on the metal grating period. The central resonance wavelength of the dip increases as $P$ increases. This is physical reasonable due to a reduced grating vector $\left(k_{\mathrm{G}}\right)$ based on Eq. (9). Fig. 5 (b) gives the corresponding variation in the sensitivity $S$ as well as $Q$ with $P$. It can be seen that $S$ increases while $Q$ decreases as the value of $P$ increases. 

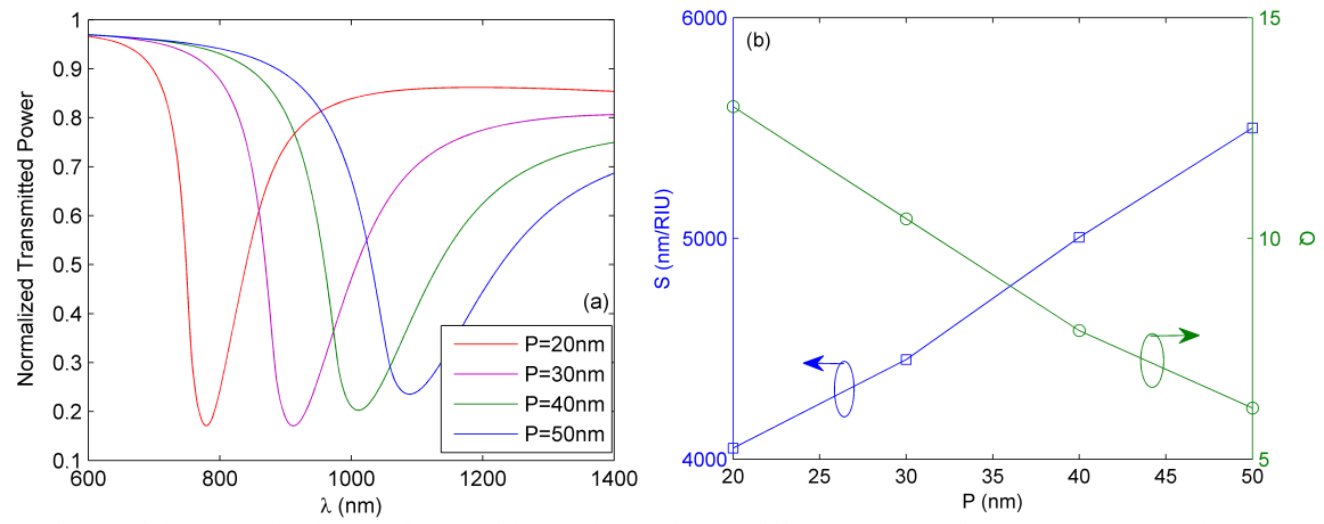

Fig. 5 (a) Variation of the normalized transmission with wavelength for the different metal grating period $P$ values. (b) Variation of the sensitivity $S$ and $Q$ at $n_{s}=1.36$ vs. the metal grating period $P$.

Fig. 6 (a) shows the normalized transmission spectra at different values of the metal grating thickness $h$. In this simulation the structural parameters are $L=20 \mathrm{~mm}, D=400 \mathrm{um}, d=10 \mathrm{~nm}, P=20 \mathrm{~nm}$, $\varepsilon_{D}=n_{c l}^{2}$ and $n_{s}=1.36$. It can also be seen that the effect of the metal grating thickness on the spectral response is clear. The central resonance wavelength as well as the transmission value of the dip decreases as $h$ decreases. This could possibly be due to the variation in the mode coupling between the surface plasmon mode and the evanescent field produced by the guided rays in fiber core. The evanescent field excites SPPs at the metal-dielectric sensing layer interface. As the metal grating layer thickness becomes thinner, evanescent fields located in the vicinity of the metal layer become stronger, as a result the coupling of evanescent field with SPPs also strengthens, hence the SPR wavelength dip shifts downwards. Fig. 6 (b) shows the corresponding variation of the sensitivity $S$ as well as $Q$ vs. $h$. It can be seen that the values of both $\mathrm{S}$ and $Q$ increase as $h$ increases.
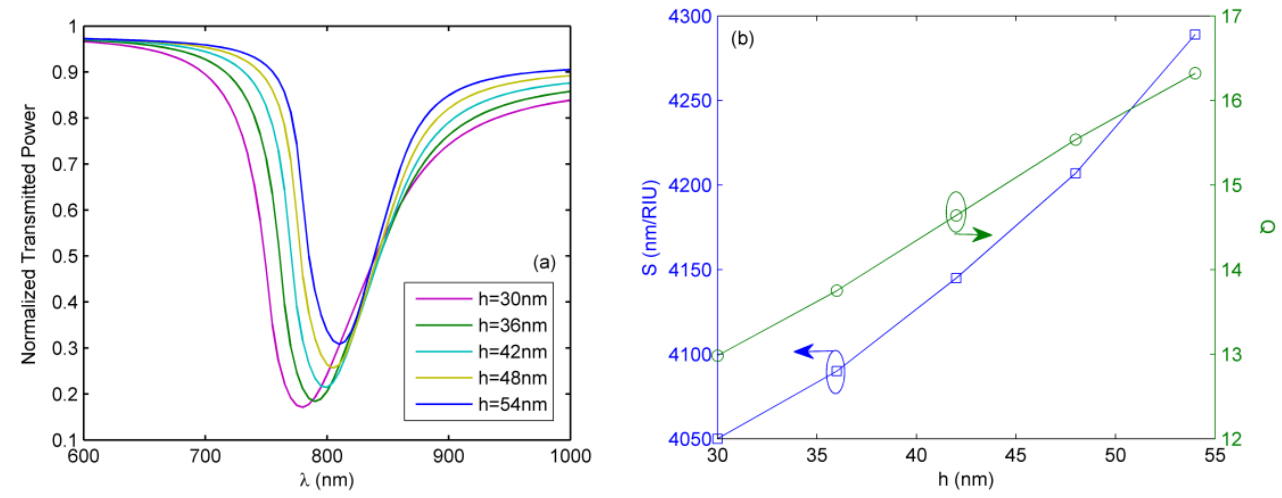

Fig. 6 (a) Variation of the normalized transmission with wavelength for the different metal grating layer thickness $h$. (b) Variation of the sensitivity $S$ and $Q$ at $n_{s}=1.36$ vs. the metal grating layer thickness $h$.

Finally Figure 7 depicts the corresponding variations in the sensitivity $S$ as well as $Q$ for different values of the grating length $L$ and the fiber core diameter $D$. For the results shown in Fig. 7 (a), the structural parameters are $h=30 \mathrm{~nm}, D=400 \mathrm{um}, d=10 \mathrm{~nm}, P=20 \mathrm{~nm}, \varepsilon_{D}=n_{c l}^{2}$ and $n_{s}=1.36$, while the structural parameters for the simulation in Fig. 7 (b) are $h=30 \mathrm{~nm}, L=20 \mathrm{~mm}, d=10 \mathrm{~nm}, P=20 \mathrm{~nm}$, $\varepsilon_{D}=n_{c l}^{2}$ and $n_{s}=1.36$. From Fig. 7, it can be seen that the value of both $S$ and $Q$ decrease as $L$ increases, while $S$ and $Q$ values increase as D increases. 

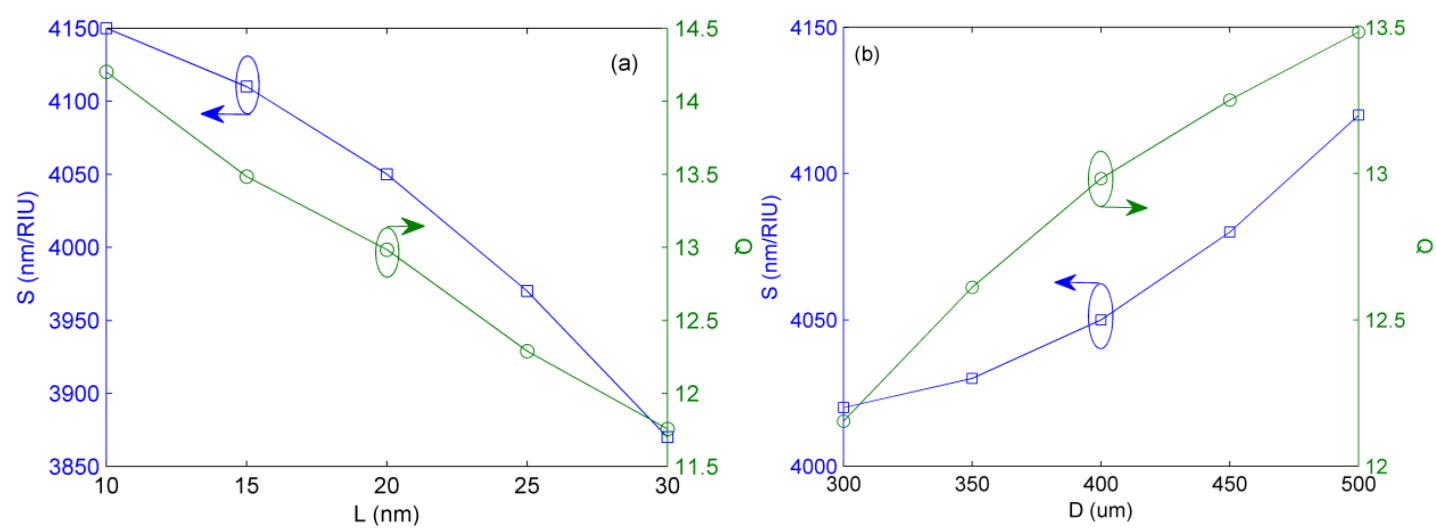

Fig. 7 Variations of the sensitivity $S$ and $Q$ at $n_{s}=1.36$ vs. the grating length $L$ (a) and the fiber core diameter $D$ (b).

Based on the results shown above, we find that the quality factor $Q$ of the SPR dip is strongly affected by the characteristic structural parameters, i.e. the fiber core diameter $D$, the grating length $L$, the metal grating period $P$ and the metal grating layer thickness $h$. However, the influence of the structural parameters of $h, D$ and $L$ on the sensitivity $S$ is not especially significant, for example for a change in $h$ from $30 \mathrm{~nm}$ to $54 \mathrm{~nm}$ the sensitivity $S$ changes by circa $6 \%$, whereas the metal grating period $P$ has a relatively larger effect with $35.8 \%$ variation in the sensitivity $S$ as $P$ varies from $20 \mathrm{~nm}$ to $50 \mathrm{~nm}$ as evident in Fig. 5 (b).

\section{Conclusions}

In conclusion, a novel fiber surface plasmon resonance biosensor with a sub-wavelength metal grating layer is numerically investigated based on a multilayer model. On the basis of the above observations, the following conclusions can be drawn. The proposed sensor has an estimated sensitivity of $4624 \mathrm{~nm} / \mathrm{RIU}$ in the RI range from 1.355 to 1.365 , which is higher than that achieved $4262 \mathrm{~nm} / \mathrm{RIU}$ (RI: $1.355-1.365$ ) in [2], and an estimated sensitivity of $13000 \mathrm{~nm} / \mathrm{RIU}$ in the RI range from 1.395 to 1.40 , which is significantly higher than that achieved $7500 \mathrm{~nm} / \mathrm{RIU}$ (RI: 1.395-1.40) in [15].The effects of the grating length, the fiber core diameter and the metal grating layer thickness on the sensitivity $S$ are not especially significant, while the grating period has relatively larger influence on the sensitivity $S$. The quality factor $Q$ of the SPR dip is strongly dependent on the grating length, the fiber core diameter, the metal grating layer thickness and the grating period.

\section{Acknowledgement}

This work was supported by Dublin Institute of Technology under the Fiosraigh Research Scholarship, Science Foundation Ireland under grant no. 11/TIDA/B2051, 07/SK/I1200, 07/SK/I1200-STTF11 and Open Fund of State Key Laboratory of Information Photonics and Optical Communications (Beijing University of Posts and Telecommunications), P. R. China.

\section{References}

[1] K. Kurihara, H. Okhawa, Y. Iwasaki, O. Niwa, T. Tobita and K. Suzuki, Fiber-optic conical microsensors for surface plasmon resonance using chemically etched single-mode fiber, Anal. Chim. Acta 523 (2004) 165-170.

[2] P. Bhatia and B. D. Gupta, Surface-plasmon-resonance-based fiber-optic refractive index sensor: sensitivity enhancement, Appl. Opt. 50 (2011) 2032-2036.

[3] B. Liedberg, C. Nylander and I. Sundstrom, Surface plasmon resonance for gas detection and biosensing, Sens. Actuators. 4 (1983) 299-304.

[4] Y. Wang, X. G. Jiang, Q. Li, Y. Wang and Z. Q. Cao, High-resolution monitoring of wavelength shifts utilizing strong spatial dispersion of guide modes, Appl. Phys. Lett. 101 (2012) 061106. 
[5] A. Abbas, M. J. Linman and Q. Cheng, New trends in instrumental design for surface plasmon resonance-based biosensors, Biosensors and Bioelectronics 26 (2011) 1815-1824.

[6] J. Albert, L. Y. Shao, C. Caucheteur, Titled fiber bragg grating sensors, Laser Photonic Rev. 7 (2013) 83-108.

[7] M. Piliarik, J. Homola, and J. Ctyroký, Surface plasmon resonance sensor based on a single-mode polarization-maintaining optical fiber, Sens. Actuators B. 90 (2003) 236-242.

[8] C. Caucheteur, C. Chen, V. Voisin, P. Berini and J. Albert, A thin metal sheath lifts the EH and HE degeneracy in the cladding mode refractometric sensitivity of optical fiber sensors, Appl. Phys. Lett. 99 (2011) 041118.

[9] R. Jha, G. Badenes, Effect of fiber core dopant concentration on the performance of surface plasmon resonance-based fiber optic sensor, Sens. Actuators A. 150 (2009) 212-217.

[10] M. Piliarik, J. Homola, Z. Man'1kova', J. C` tyroky', Surface plasmon resonance sensor based on a single-mode polarization-maintaining optical fiber, Sens. Actuators B. 90 (2003) 236-242.

[11]S. K. Mishra, D. Kumari, B. D. Gupta, Surface plasmon resonance based fiber optic ammonia gas sensor using ITO and polyaniline, Sens. Actuators B. 171-172 (2012) 976-983.

[12] A. K. Sharma and B.D. Gupta, On the sensitivity and signal to noise ratio of a step-index fiber optic surface plasmon resonance sensor with bimetallic layers, Opt. Commun. 245 (2005) 159-169.

[13] A. K. Sharma and B.D. Gupta, Fiber optic sensor based on surface plasmon resonance with nanoparticle films, Photonics and Nanostructures: Fundamentals and Applications. 3 (2005) 30-37.

[14] A. Hosoki, M. Nishiyama, H. Lgawa, A. Seki, Y. Choi and K. Watanabe, A surface plasmon resonance hydrogen sensor using $\mathrm{Au} / \mathrm{Ta}_{2} \mathrm{O}_{5} / \mathrm{Pd}$ multi-layers on hetero-core optical fiber structures, Sens. Actuators B. 185 (2013) 53-58.

[15] Karla Balaa, Malak Kanso, Stephane Cuenot, Tiberiu Minea, Guy Louarn, Experimental realization and numerical simulation of wavelength-modulated fibre optic sensor based on surface plasmon resonance, Sen. Actuators B. 126 (2007) 198-203.

[16] Yinquan Yuan, Liyun Ding, Zhenqiang Guo, Numerical investigation for SPR-based optical fiber sensor, Sen. Actuators B: Chemical, 157 (1) (2011) 240-245.

[17] C. W. Haggans and L. F. Li, Effective-medium theory of zeroth-order lamellar gratings in conical mountings, J. Opt. Soc. Am. A. 10 (1993) 2217-2225.

[18] T. H. Isaac, W. L. Barnes and E. Hendry, Determining the terahertz optical properties of subwavelength films using semiconductor surface plasmons, Appl. Phys. Lett. 93 (2008) 241115.

[21] Y. X, A. cottenden and N. B. Jones, An approximate theoretical model of surface plasmon resonance optical waveguide and fibre-optic sensors, Opt. Quant. Electron. 37 (2005) 1129-1140.

[19] Y.S. Dwivedi, A.K. Sharma, B.D. Gupta, Influence of design parameters on the performance of a surface plasmon sensor based fiber optic sensor, Plasmonics 3 (2008) 79-86. 